\title{
Non-contact ultrasonic resonant spectroscopy resolves the elastic properties of layered plant tissues
}

\author{
T. E. G. Álvarez-Arenas, ${ }^{1, a)}$ D. Sancho-Knapik, ${ }^{2}$ J. J. Peguero-Pina, ${ }^{2}$ A. Gómez-Arroyo, ${ }^{2}$ \\ and E. Gil-Pelegrín ${ }^{2}$ \\ ${ }^{1}$ ITEFI-CSIC, Serrano 144, 28006 Madrid, Spain \\ ${ }^{2}$ CITA de Aragón, Montañana 930, 50059 Zaragoza, Spain
}

(Received 5 October 2018; accepted 5 December 2018; published online 21 December 2018)

\begin{abstract}
This paper describes the application of the wide-band non-contact ultrasonic resonant spectroscopy technique to layered plant tissues (leaves), a method to extract the properties of main component tissues: palisade parenchyma and spongy mesophyll, a verification of the obtained properties, and a discussion of the implications of the observed elastic anisotropy. Transmission coefficient spectra of Ligustrum lucidum leaves with the thickness in the range of $250-850 \mu \mathrm{m}$ revealing several order thickness resonances have been measured. A leaf acoustic model based on a two-layered structure and a metaheuristic (simulated annealing algorithm) is used to solve the inverse problem. The extracted parameters of these two layers of tissue are consistent with cross-sectional cryo-SEM images and other independent measurements. The extracted resonant frequency and the impedance of each layer explain the origin of the observed resonances. Finally, the elastic modulus of each layer is extracted and analyzed. The presented technique is a unique tool to study (in vivo and in a completely non-invasive way) the ultrasonic, elastic, and viscoelastic properties of layered plant tissues which could lead to a better understanding of the relationship between the tissue microstructure and the tissue function with macroscopic properties and how this may affect water relations. Published by AIP Publishing. https://doi.org/10.1063/1.5064517
\end{abstract}

The non-contact resonant ultrasonic spectroscopy (NC-RUS), limited to the first thickness resonance, was first applied to plant leaves in Ref. 1. The leaf total thickness $\left(t_{\text {TOTAL }}\right)$, effective density $\left(\rho_{\text {eff }}\right)$, ultrasound velocity $\left(v_{\text {eff }}\right)$, and attenuation $\left(\alpha_{\text {eff }}\right)$ were extracted by solving the inverse problem (IP) defined by the NC-RUS measurements and a simplified leaf acoustic model: a homogeneous and flat layer. $^{2}$ Later, Ref. 3 revealed a close relationship between the NC-RUS parameters and the leaf turgor pressure that allowed determining the turgor loss point. Moreover, this technique provided a unique tool to study the elastic properties of plant tissues ${ }^{4}$ and the in-vivo leaf response to environmental stimuli. ${ }^{5}$

In most cases, a wider frequency range reveals several orders of thickness resonances. However, these resonances normally present a significant harmonic distortion that makes impossible to apply the simplified model mentioned previously. This distortion is suspected to be due to the layered leaf structure (palisade parenchyma-PP, spongy mesophyll-SM, and epidermises). ${ }^{6,7}$ If confirmed, the analysis of these resonances would make possible to obtain information about the component layers in a non-invasive and in-vivo way, which is nowadays not feasible. However, this possibility was hindered by two major hurdles. (1) The lack of an efficient and robust solution of the IP for layered plant tissues. (2) The impossibility to confirm extracted parameters by using either independent data or well-controlled samples. This work focuses on the solution of these problems, on the interpretation of the measured spectra under this perspective, and on the analysis of the extracted information of the leaf constituent layers.

\footnotetext{
a)Author to whom correspondence should be addressed: t.gomez@csic.es
}

For layered plates, the solution of the IP provides four parameters for each sublayer ${ }^{8}$

$$
\left\{p_{i}\right\}=\left\{f_{i}^{0}, Z_{i}, \alpha_{i} t_{i}, n_{i}\right\}, i=1, \ldots n, n \equiv \text { number of layers }
$$

where $f_{i}^{0}=v_{i} / t_{i}$ ( $t$ : thickness), $Z$ is the acoustic impedance, and $n$ is given by ${ }^{9}$

$$
\alpha=\alpha_{0}\left(f / f_{0}\right)^{n}
$$

where $f$ is the frequency and $\alpha_{0}$ is the attenuation at $f_{0}$. It is worthwhile noting that the resonant frequencies of a layer separating two media $(0$ and $F)$ appear at either $[(n+1) / 2] f_{i}^{0}$ or $[(2 n+1) / 4] f_{i}^{0}$, where $\mathrm{n}=0,1,2, \ldots$ The former are $\lambda / 2$ resonances and correspond to $\mathrm{Z}_{0}<\mathrm{Z}_{\mathrm{LAYER}}>\mathrm{Z}_{\mathrm{F}}$ or $\mathrm{Z}_{0}>\mathrm{Z}_{\mathrm{LAYER}}$ $<Z_{\mathrm{F}}$, while the latter are $\lambda / 4$ resonances that correspond to $Z_{0}$ $<\mathrm{Z}_{\text {LAYER }}<\mathrm{Z}_{\mathrm{F}}$ or $\mathrm{Z}_{0}>\mathrm{Z}_{\mathrm{LAYER}}>\mathrm{Z}_{\mathrm{F}}$.

In addition, the surface density of each layer, $\rho_{S U P}^{i}$, is given by $\left(Z_{i} / f_{i}^{0}\right)$ and the effective surface density of the whole leaf $\left(\rho_{S U P}^{e f f}\right)$ is obtained using the following equation:

$$
\rho_{S U P}^{e f f}=\sum_{i} \rho_{S U P}^{i}=\sum_{i}\left(\frac{Z_{i}}{v_{i} / t_{i}}\right) .
$$

It is clear then that for plant leaves, it is not feasible or at least extremely difficult to obtain alternative data for $\left\{p_{i}\right\}$ by using either alternative techniques or existing available data, the only exception being $\rho_{S U P}^{\text {eff }}$.

The technique proposed in Ref. 10 also allows us to

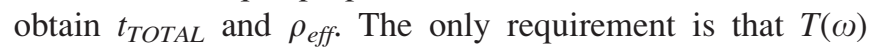
is measured in a frequency range large enough, so that it provides a low frequency range where several orders of 
thickness resonances are observed plus a high frequency range where the effect of these resonances on the phase measurement can be considered negligible.

Given the NC-RUS experimental set-up, ${ }^{1,11,12}$ the measured phase $\theta$ is given by Eq. (4)

$$
\theta=\operatorname{angle}\left(\frac{F F T\left(S_{S A M P}\right)}{F F T\left(S_{C A L}\right)}\right),
$$

where $\left(\mathrm{S}_{\mathrm{CAL}}\right)$ is the through transmitted signal between two transducers without sample in-between and $\mathrm{S}_{\text {SAMP }}$ is the signal with the sample at normal incidence.

At the high frequency limit, $\theta=\omega\left(\right.$ tof $\left.f^{S A M P}-t^{C A L}\right)$, where tof ${ }^{S A M P}$ and $t o f^{C A L}$ are the time of flight of $S_{S A M P}$ and $S_{C A L}$, respectively. It is straightforward to prove that

$$
\theta=-\frac{t_{\text {TOTAL }}}{v_{0}}+\sum_{i} \frac{t_{i}}{v_{i}},
$$

where $v_{0}$ is the ultrasound velocity in the surrounding fluid. As $v_{0}$ is known, $\theta$ is a direct measurement, and $t_{i} / v_{i}$ are extracted from solving the IP, we can then work out $t_{\text {TOTAL }}$ and hence $\rho_{\text {eff }}$ can also be worked out. These two parameters allow for a direct comparison with independent measurements. In this case, the parameters extracted are $\left\{p_{i}^{*}\right\}=\left\{f_{i}^{0}, Z_{i}, \alpha_{i} t_{i}, n_{i}\right.$, $\left.\rho_{\text {SUP }}^{i}, \rho_{\text {SUP }}^{\text {eff }}, t_{\text {TOTAL }}, \rho_{\text {eff }}\right\}, i=1, \ldots n, n \equiv$ number of layers.

The number of layers in the model is selected following Occam's razor principle according to two main ideas: (i) the model should resemble the actual leaf structure well enough so that the extracted parameters relate to the actual leaf properties and (ii) it must be simple enough so that the inverse problem is tractable. Leaves may have a complex structure, in Ligustrum lucidum leaves, and ignoring the vascular structure, six main layers can be distinguished (1. Cuticle, 2. Adaxial epidermis, 3. Palisade parenchyma, 4. Spongy mesophyll, 5. Abaxial epidermis, and 6. Cuticle); however, such a model, if no additional information is provided, leads to an intractable IP. Fortunately, it can be largely simplified as cuticles are very thin and must have a negligible ultrasonic effect. Moreover, epidermises are also thin (« wavelength) and their effect could also be considered negligible. This is especially true for the adaxial epidermis as the difference between the impedances of this epidermis and the adjacent PP layer must be very small. The presence of abaxial epidermis may be more significant, although its thickness is still much smaller than the wavelength. However, the presence of stoma (through holes in the epidermis for gas transfer) makes this layer more transparent to the ultrasonic wave. So, it seems reasonable to start with a layered acoustic model for the leaf with two sublayers. One of these layers of the model corresponds to adaxial epidermis $+\mathrm{PP}$, while the other corresponds to $\mathrm{SM}+$ abaxial epidermis. This model resembles the main acoustic differences in the actual leaf (which is due to the huge porosity and cell structure differences between PP and SM), while giving rise to an IP solvable without the aid of any additional information. The capability of this model to reproduce the behavior of all measured leaves will eventually determine its suitability. Clearly, the model to be used depends on the species under study; for some other species, a sandwich acoustic leaf model with three layers (but with the same complexity as two of them are identical) has already been proposed (see Ref. 7).

The two-layer model has also the advantage that if the ratio (r) $\mathrm{r}=t_{1} / t_{2}$ is determined by any independent available method (e.g., analysis of leaf cross-sectional images), it is possible to convert $\left\{p_{i}^{*}\right\}$ into $\left\{\tilde{p}_{i}\right\}=\left\{M_{i}, \rho_{i}, \alpha_{i}, n_{i}, t_{i}\right\}$, $i=1, \ldots 2$, where $M$ is the elastic modulus defined as $M=v^{2} \rho$. The main advantage of $\left\{\tilde{p}_{i}\right\}$ over $\left\{p_{i}^{*}\right\}$ or $\left\{p_{i}\right\}$ is that there is a considerable amount of information about the elastic modulus of plant tissues and cellular solids and that the interpretation of $M$ in terms of the features observed in leaf cross-sectional images (porosity, cell shape, cell wall thickness, etc.) is easier than it is for $Z$.

In addition, and with the purpose of identifying cases of study that could help to determine the correctness of the extracted parameters, our group has been working over the past few years on the application of the NC-RUS technique to different dicot species in parallel with the study of the leaf tissue structure by using cryo-SEM, TEM, and optical microscopy. This work provided a very interesting candidate for this purpose: the leaves of Ligustrum lucidum. This species presents leaves with a large thickness variation (250-900 $\mu \mathrm{m})$, which is largely due to the variation in one parameter: the thickness of the PP layer.

Having a sensible solution to the verification problem, we can focus on the IP. The simplified two-layer leaf acoustic-model, to calculate $T(\omega)$ for any given combination of $\left\{p_{i}\right\} \equiv v_{i} / t_{i}, Z_{i}, \alpha_{i} t_{i}, n_{i}$, and a metaheuristic approach [in particular, the Simulated Annealing (SA) algorithm] were implemented. $\left\{p_{i}\right\}$ are then extracted by solving the IP: maximizing the fitting of the theoretically calculated spectra$|T(\omega)|$ and $\phi[T(\omega)]$ - to the measured ones. The SA approach is selected for its capability to deal with the high dimensionality of this problem dimension $=8$ and to find optimum values even in the presence of local maxima. The SA algorithm is basically a Monte Carlo method, where sampling is performed by a Markov chain and an acceptance criterion is given by the Metropolis algorithm together with a cooling scheme that determines the initial temperature, the length of the homogeneous Markov chains, and the temperature decrease. ${ }^{13,14}$ The algorithm was implemented in Python 2.7; for each measurement, the algorithm is run 6 times.

Two pairs of air-coupled transducers, designed and built at CSIC, were used for the measurements with the purpose of covering a frequency band large enough. Centre frequencies are $300 \mathrm{kHz}$ (bandwidth: $180-425 \mathrm{kHz}$ ) and $800 \mathrm{kHz}$ (bandwidth: $0.4-1.2 \mathrm{MHz}$ ) and peak sensitivities are -30 and $-35 \mathrm{~dB}$, respectively. Transducers were mounted on $\mathrm{U}-$ shaped holders that provide correct positioning of both transducers and a slot in the center of the cavity that is used to correctly place the leaves. ${ }^{10}$ Figure 1 shows a picture of the device and a schematic view of the design. The rest of the equipment (pulser/receiver and oscilloscope) and the measurement procedure are explained in Refs. 1, 10, and 11.

Leaves without defects were harvested at dawn, transported to the lab, preserved at full turgor and measured that same morning. A set of 18 leaves were used for NC-RUS measurements, each one measured at two different locations (see Fig. 1). Once measured, a $24 \mathrm{~mm}$ diameter circle was cut to measure the thickness and the mass and work out 


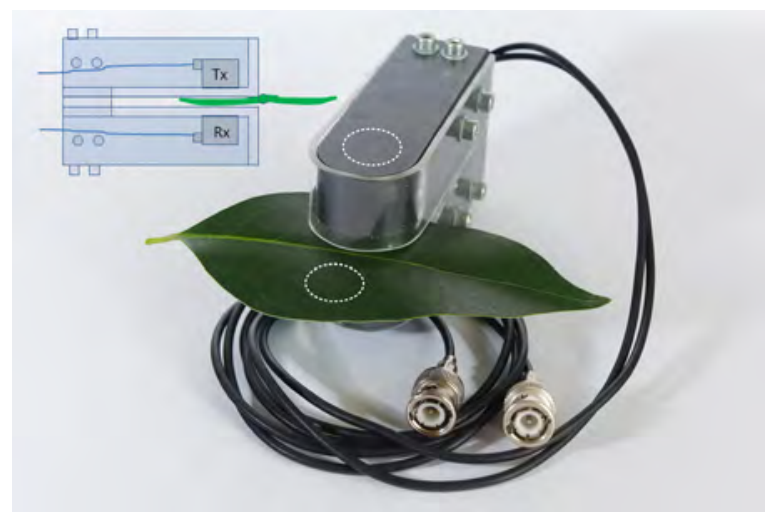

FIG. 1. Picture of the device used for the measurements: a U-shaped holder for the transducers (Tx and Rx) and a polycarbonate cover that provides a slot to properly place the leaf for the measurement. The two white dashed circle lines indicate the location of the transducer in the holder and the size and the position of the area where measurements were performed on both sides of the midrib (only one is indicated).

surface and volumetric densities. Other 31 samples were used only for thickness, surface density, and volumetric density measurements. Out of this set, a smaller group 4 samples was selected for cryo-SEM analysis of the cross-section. Finally, 21 leaves were used to study the relationship between $t_{\text {TOTAL }}$ and the thickness ratio of palisade parenchyma to spongy mesophyll $\left(t_{P P} / t_{S M}\right)$. In this case, each leaf was cut into two cross-sections $(0.2-0.3 \mathrm{~mm}$ apart) with a scalpel and the aid of a dissecting microscope. A light microscope was used to capture pictures of the mesophyll cross-section which were later analyzed using ImageJ to obtain $t_{P P} / t_{S M}$ vs $t_{T O T A L}$. Equation (6) shows the obtained relation from the linear regression analysis of these data $(\mathrm{R}$-squared $=0.904)$

$$
\frac{t_{P P}}{t_{S M}}=3.404 \mathrm{~mm}^{-1} t_{T O T A L}+0.0719 .
$$

Figure 2 presents two cryo-SEM images of the cross-section of two leaves.

Figure 3 illustrates the variation in $T(\omega)$ with $t_{T O T A L}$ by showing four cases. Figure 3 also shows the calculated spectra (using the extracted values of $\left\{p_{i}\right\}$ by the SA algorithm). The remarkable goodness of this fitting reveals both the efficiency of the SA algorithm and that the two-layer leaf acoustic-model provides a faithful representation of the leaf ultrasonic response.

Let us first verify some of the global leaf parameters $\left(\rho_{\text {eff }}, t_{\text {TOTAL }}\right.$ and $\left.\rho_{\text {SUP }}^{\text {eff }}\right)$ extracted by NC-RUS by comparing them with data obtained by conventional (conv) means. The linear regression analysis of $\rho_{\text {eff }}^{N C-R U S}$ vs $\rho_{\text {eff }}^{\text {Conv }}$ revealed

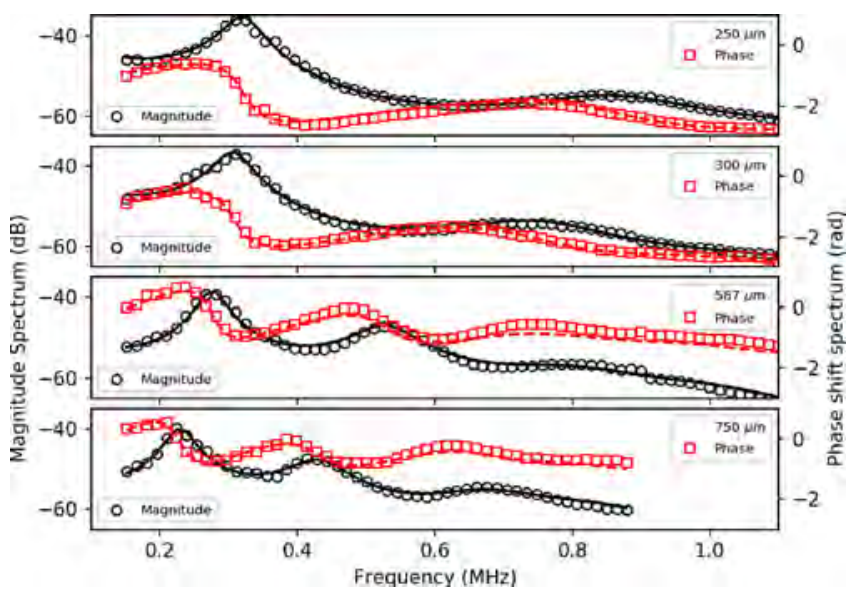

FIG. 3. Magnitude and phase spectra of the transmission coefficient of four leaves. Experimental data and calculated ones (solid line) using a two-layer model and the layer parameters extracted by the solution of the IP.

$\rho_{\text {eff }}^{N C-R U S}=0.94 \rho_{\text {eff }}^{\text {Conv }}+33 \mathrm{~kg} / \mathrm{m}^{3}$, with R-squared $=0.988$. That is, pretty close to the diagonal. While for $\rho_{S U P}^{\text {eff }} v s t_{T O T A L}$, we obtained

$$
\begin{aligned}
\rho_{S U P}^{\text {eff }}\left(\frac{\mathrm{kg}}{\mathrm{m}^{2}}\right) & =8.6 \times 10^{-4} t_{\text {TOTAL }}(\mu \mathrm{m})+7.5 \times 10^{-5}, \\
R^{2} & =0.981, N C-R U S \\
\rho_{\text {SUP }}^{\text {eff }}\left(\frac{\mathrm{kg}}{\mathrm{m}^{2}}\right) & =9.78 \times 10^{-4} t_{\text {TOTAL }}(\mu \mathrm{m})-3.8 \times 10^{-2}, \\
R^{2} & =0.989, \text { conventional. }
\end{aligned}
$$

Therefore, the agreement between NC-RUS and conventional measurements is quite good in all cases. Let us analyze some of the extracted parameters for the individual sublayers in the model: PP and SM layers. We first focus on $f$ and $Z$ (Fig. 4).

Figure 4 shows that $f_{P P}$ decreases as $t_{T O T A L}$ increases. This is an expected result as we know that $f_{P P}=v_{P P} / t_{P P}$ and that $t_{P P}$ notably increases when the leaf becomes thicker. In particular, when $t_{\text {TOTAL }}$ varies from 340 to $780 \mu \mathrm{m}, t_{P P}$ increases by a factor of $\sim 2.75$ (Fig. 2), while $f_{P P}$ increases by a factor of $\sim 2.99$. That is, the variation in $f_{P P}$ can be, in great part, explained by the increase in $t_{P P}$. On the contrary, $f_{S M}\left(t_{T O T A L}\right) \cong c t e$. As cryo-SEM images show that $t_{S M}$ slightly increases with $t_{T O T A L}, v_{S M}$ must then increase in a similar proportion.

Figure 4 shows that $Z_{A I R}<Z_{S M}<Z_{P P}$ and that $Z_{S M}<Z_{P P}>Z_{A I R}$. We can try the simplistic interpretation previously mentioned and consider $T$ composed of two series
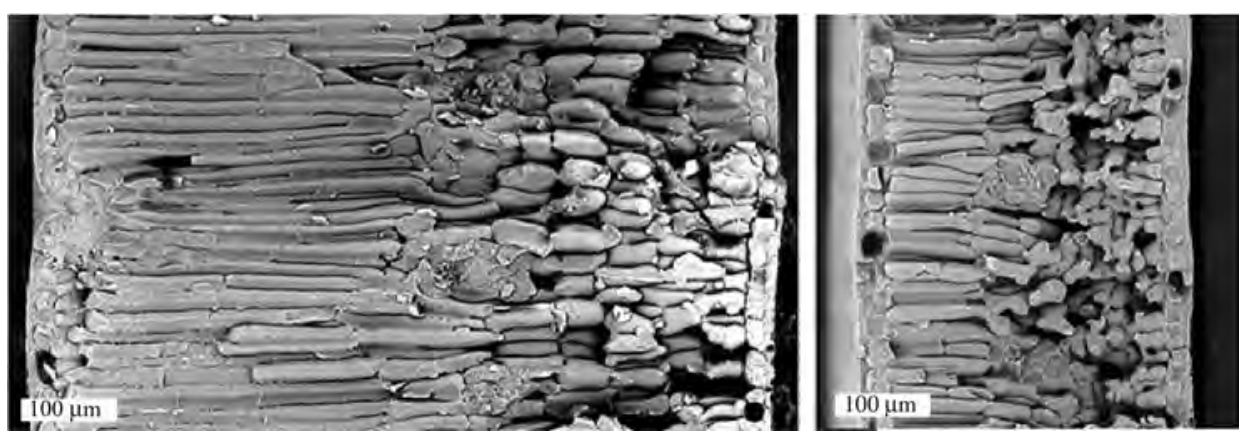

FIG. 2. Cryo-SEM micrograph of two Ligustrum lucidum leaves. (Total leaf thickness, left: $780 \mu \mathrm{m}$, right: $340 \mu \mathrm{m}$.) 


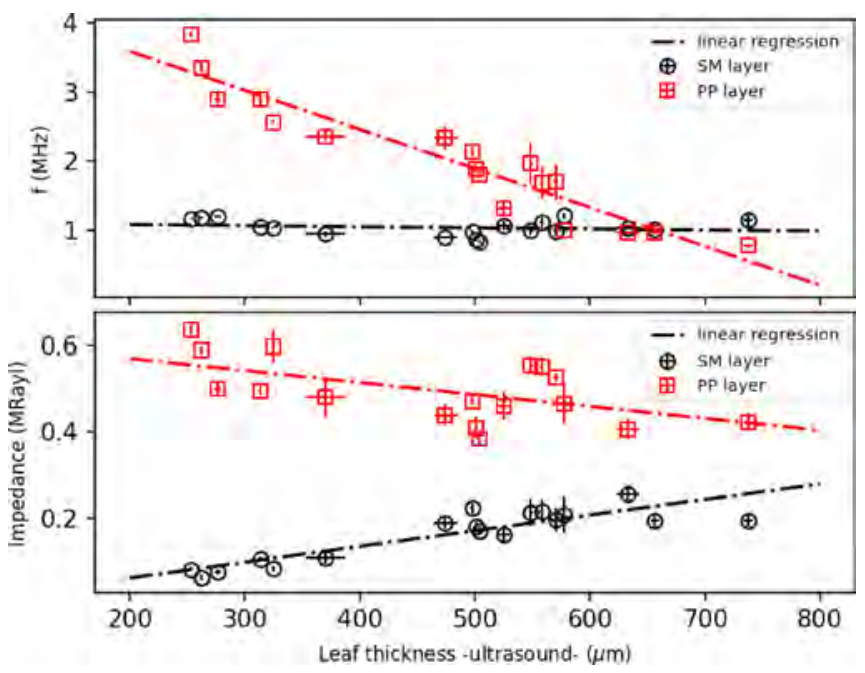

FIG. 4. Variation of $\mathrm{f}$ and $\mathrm{Z}$ with the total leaf thickness. A linear regression is also included just for the sake of making more evident the observed trends.

of resonances: a $\lambda / 4$ series due to the SM layer and a $\lambda / 2$ series due to the PP layer. The $250 \mu \mathrm{m}$ leaf (Fig. 3) presents two resonances, at 0.32 and $0.86 \mathrm{MHz}$. From Fig. 4, we know that $f_{S M} \approx 1.21 \mathrm{MHz}$, that is, the first two orders of the ideal $\lambda / 4$ SM-resonances are expected at 0.30 and $0.91 \mathrm{MHz}$. On the other hand, $f_{P P} \approx 3.86 \mathrm{MHz}$; therefore, the first $\lambda / 2$ PP-resonance is expected at $1.93 \mathrm{MHz}$. This suggests that the two resonances in Fig. 3 mainly correspond to the first two orders of $\lambda / 4$ SM-resonances that appear slightly displaced away from the $\lambda / 4$ values. Moreover, the $\lambda / 2$ PP-resonances are not observed because they are expected at frequencies beyond the experimental range and are, most likely, completely damped out. This frequency shift is produced by the fact that in the relationship $Z_{A I R}<Z_{S M}<Z_{P P}$, PP is not a semi-infinite medium, but a finite thickness layer, so the SM layer is only partially loaded by the PP. To understand this effect, we can consider what happens in the limit case, when the PP layer becomes so thin that it is negligible. In this case, we have $Z_{A I R}<Z_{S M}>Z_{A I R}$, and then, the resonance of the SM layer will be shifted up to the $\lambda / 2$ value. This feature is of importance because it denotes that the transmission coefficient contains information about the PP layer in the resonance of the SM layer even when the resonances of the PP layer are not observed.

As $t_{\text {TOTAL }}$ increases beyond $\sim 500 \mu \mathrm{m}$, there appears a third resonance (Fig. 3). We know that $f_{P P}$ decreases with $t_{T O T A L}$, so this third resonance can be due to the appearance of $f_{P P}$ within the experimental frequency band. For example, for a $587 \mu \mathrm{m}$ leaf, $f_{S M} \approx 1.24 \mathrm{MHz}$ and $f_{P P} \approx 1.1 \mathrm{MHz}$ (Fig. 4); hence, the first two $\lambda / 4$ SM-resonances are expected at 0.31 and $0.93 \mathrm{MHz}$ and the first two $\lambda / 2 \mathrm{PP}$-resonances are expected at 0.55 and $1.1 \mathrm{MHz}$. The observed resonances in Fig. 3 are located at $0.28,0.53$, and $0.81 \mathrm{MHz}$. The first and third resonances are close to the first and second order $\lambda / 4$ SM-resonances, while the second is close to the first order $\lambda / 2$ PP-resonance. Deviation of actual values away from the $\lambda / 4$ or $\lambda / 2$ values is due to the non-negligible mutual influence of both layers and the coupling of the resonances.

The fact that $Z_{S M}<Z_{P P}$ is an expected result, as SM presents a much larger porosity $\left(\rho_{S M}<\rho_{P P}\right)$ and a more rounded cell geometry with more free spaces (hence $\left.v_{S M}<v_{P P}\right) .{ }^{15}$ These different tissue properties are not specific to Ligustrum lucidum leaves and are common in most species as they have their origin in different functions these tissues play (gas change in SM and interaction with light in PP).

The attenuation coefficient and its variation with the frequency [Eq. (2)] are also extracted by the solution of the IP, although the analysis of these parameters exceeds the scope of this paper, it can be mentioned that the attenuation coefficient at $400 \mathrm{kHz}$ in PP decreases with the PP thickness and varies from $1000 \mathrm{~Np} / \mathrm{m}$ to $546 \mathrm{~Np} / \mathrm{m}$, while it is rather constant in SM and about $1140 \mathrm{~Np} / \mathrm{m}$. $n$ [Eq. (2)] in SM is rather constant $\sim 2.0$, while in $\mathrm{PP}$, it increases slightly from 0.6 to 1.5 .

Taking into consideration the information in Eq. (6), we can derive $\left\{\tilde{p}_{i}\right\}$ from $\left\{p_{i}^{*}\right\}$. In particular, we focus here on the analysis of the elastic modulus $(M)$. The results are shown in Fig. 5.

The larger value of $\mathrm{M}_{\mathrm{PP}}$ was expected from the PP cellular structure: cells well packed and elongated in the thickness direction. $\mathrm{M}_{\mathrm{SM}}$ presents very low values that are consistent with the large porosity and the rounded cellular shape. The increase in $\mathrm{M}_{\mathrm{SM}}$ with $\mathrm{t}_{\mathrm{TOTAL}}$ can be due to the tendency of having cells that become more elongated in the thickness direction as $t_{\text {TOTAL }}$ increases (Fig. 2). These elastic moduli can be compared with data currently obtained by different methods in plant physiology; however, it must be underlined that the value obtained with this technique is inherently different for three main reasons: (i) available methods do provide an overall leaf-effective modulus and cannot resolve different tissues, (ii) unlike many other methods in plant physiology, where the strain induced to study the elastic response of the tissues and cells is quite large (indentation: 0.01-0.1; Scholander camera: $5 \times 10^{-3}-5 \times 10^{-2}$ ), the strain produced by NC-RUS is much smaller (because the efficiency of air-coupled ultrasound is very limited, with peak pressure output of air-coupled transmitters around $1 \mathrm{kPa}^{16}$ ) in the range of $\sim 2 \times 10^{-6}-2.0 \times 10^{-5}$. Being so small that it is expected that $\mathrm{M}$ is measured in the linear range and (iii) the deformation rate in NC-RUS is so fast (in the low $\mathrm{MHz}$ region) that the cell deformation takes place at constant fluid

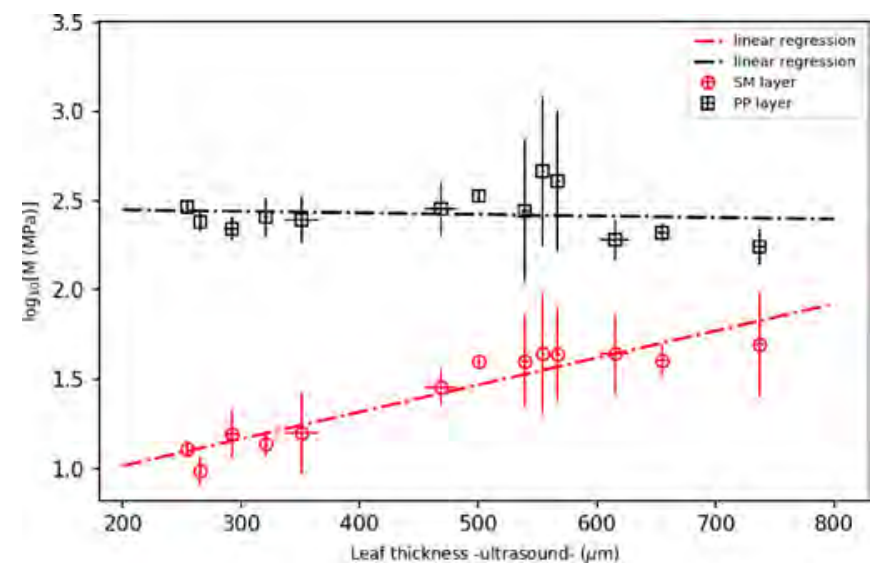

FIG. 5. Variation in the elastic modulus $\boldsymbol{M}$ with $t_{T O T A L}$ for $S M$ and $P P$ layers. 
content as it is beyond the poroelastic relaxation time which can be estimated in the range of $0.1-5.0 \mathrm{~s} .{ }^{17} \mathrm{~A}$ very interesting result is the fact that $\mathrm{M}_{\mathrm{PP}} \gg \mathrm{M}_{\mathrm{SM}}$; this may lead to significant differences in the behavior of the turgor pressure in PP and SM. This will surely influence the evolution and the equilibrium of the global leaf water potential. This feature will be investigated in the future.

In conclusion, a procedure to apply wide-band NCRUS to plant leaves and to extract information about the different constituent layers has been presented. It has been applied to Ligustrum lucidum leaves. The fitting of the calculated transmission coefficient by the leaf acoustic layered model with two sublayers to the experimental data is excellent, revealing both that the model is a sensible representation of the actual leaf and the efficiency of the SA algorithm. The total thickness and surface density obtained from the NC-RUS technique have been compared with results provided by conventional methods revealing very good agreement which means a first validation of the technique. Moreover, the extracted thickness resonant frequency and impedance for the two layers of tissue provided an explanation of the origin of the observed resonances. Finally, and with the aid of independent $t_{\mathrm{PP}} / \mathrm{t}_{\mathrm{SM}}$ ratio measurements, it was possible to obtain $\mathrm{M}$, which revealed relatively low values, consistent with the cellular structure of these tissues, and significant differences between $M_{P P}$ and $\mathrm{M}_{\mathrm{SM}}$. It is suggested that this difference may produce an imbalance of the turgor pressure and then play a role in the water behavior in the leaf that should be considered in the future to better understand the leaf response to water potential variations.
The support from the Spanish State Research Agency and European Regional Development Fund (ERDF/FEDER), Grant No. DPI2016-78876-R, is acknowledged.

${ }^{1}$ T. E. G. Alvarez-Arenas, D. Sancho-Knapik, J. J. Peguero-Pina, and E. Gil-Pelegrín, Appl. Phys. Lett. 95, 193702 (2009).

${ }^{2}$ T. E. G. Alvarez-Arenas, Ultrasonics 50, 104 (2010).

${ }^{3}$ D. Sancho-Knapik, T. E. G. Álvarez-Arenas, J. J. Peguero-Pina, and E. Gil-Pelegrín, J. Exp. Bot. 61, 1385 (2010).

${ }^{4}$ D. Sancho-Knapik, T. E. G. Álvarez-Arenas, J. J. Peguero-Pina, V. Fernández, and E. Gil-Pelegrín, J. Exp. Bot. 62, 3637 (2011).

${ }^{5}$ M. D. Fariñas, D. Sancho Knapik, J. J. Peguero Pina, E. Gil-Pelegrin, and T. E. G. Álvarez-Arenas, Ultrasound Med. Biol. 40, 2183 (2014).

${ }^{6}$ M. D. Farinãs, D. Sancho-Knapik, J. J. Peguero-Pina, E. Gil-Pelegrín, and T. E. G. Alvarez-Arenas, Appl. Phys. Lett. 102, 103702 (2013).

${ }^{7}$ M. D. Fariñas and T. E. G. Álvarez-Arenas, J. Mech. Behav. Biomed. Mater. 39, 304-315 (2014).

${ }^{8}$ L. Brekhovskikh, Waves in Layered Media (Academic Press, 1976).

${ }^{9}$ T. L. Szabo and J. Wu, J. Acoust. Soc. Am. 107(5), 2437-2446 (2000).

${ }^{10}$ T. E. G. Álvarez-Arenas, "Simultaneous thickness and ultrasonic velocity estimation in soft layered tissues," Ultrasonics (unpublished).

${ }^{11}$ T. E. G. Álvarez-Arenas, E. Gil-Pelegrín, J. Ealo Cuello, M. D. Fariñas, D. Sancho-Knapik, D. A. Collazos Burbano, and J. J. Peguero-Pina, Sensors 16(7), 1-20 (2016).

${ }^{12}$ D. Sancho-Knapik, H. Calás, J. J. Peguero-Pina, A. Ramos Fernández, E. Gil-Pelegrín, and T. E. G. Álvarez-Arenas, IEEE Trans. Ultrason. Ferroelectr. Freq. Control 59(2), 319 (2012).

${ }^{13}$ J. Dréo, P. Siarry, A. Pétrowski, and E. Taillard, Metaheuristics for Hard Optimization (Springer, 2006).

${ }^{14}$ K.-L. Du and M. N. Swamy, Search and Optimization by Metaheuristics (Birkhäuser, 2016).

${ }^{15}$ L. Gibson and M. Ashby, Cellular Solids: Structure and Properties, Cambridge Solid State Science Series (Cambridge University Press, Cambridge, 1997).

${ }^{16}$ Ł. Ambroziński, I. Pelivanov, S. Song, S. J. Yoon, D. Li, L. Gao, T. T. Shen, R. K. Wang, and M. O’Donnell, Appl. Phys. Lett. 109(4), 043701 (2016).

${ }^{17}$ J. Dumais and Y. Forterre, Annu. Rev. Fluid Mech. 44, 453-478 (2012). 